\title{
The effect of GH treatment on serum FGF23 and Klotho in GH-deficient children
}

\author{
Alexandra Efthymiadou, Dimitra Kritikou, Stefanos Mantagos \\ and Dionisios Chrysis
}

Division of Endocrinology, Department of Pediatrics, Medical School, University of Patras, Rio 26504, Patras, Greece
Correspondence should be addressed to A Efthymiadou Email

eythymiadou@upatras.gr

\begin{abstract}
Background: Normal phosphate homeostasis is essential for normal linear growth. The phosphaturic fibroblast growth factor 23 (FGF23)/Klotho axis is a major regulator of phosphate homeostasis; therefore, an intact FGF23/Klotho axis is important for normal linear growth. On the other hand, GH/IGF1 axis has opposing effects on phosphate homeostasis, but the underline mechanisms remain unclear.

Aim: The main objective of this study was to investigate the possible interactions of FGF23 and its co-receptor Klotho, with growth hormone (GH)/IGF1 axis in the regulation of phosphate metabolism in GH-deficient children under GH treatment. Methods: We studied $23 \mathrm{GH}$-deficient children, before and 3 months after the onset of GH treatment. Anthropometry and assessment of biochemical parameters were performed, as well as measurement of FGF23 (intact FGF23/iFGF23 and C-terminal FGF23/cFGF23) and soluble $\alpha$-Klotho (sKlotho) levels.

Results: After 3 months on GH treatment, the elevation of serum phosphate and TmPO4/GFR $(P<0.0001$ and $P<0.01$ respectively) was accompanied by a significant increase in CFGF23 $(P<0.01)$, iFGF23 $(P<0.0001)$, sKlotho $(P<0.0001)$ and IGF1 $(P<0.0001)$. Serum phosphate and TmPO4/GFR were positively associated with iFGF23 $(P<0.01$ and $P<0.05)$ and IGF1 $(P<0.05$ and $P<0.05)$. iFGF23 levels were positively correlated with sKlotho $(P<0.001)$, IGF1 $(P<0.0001)$ and height SDS $(P<0.0001)$, whereas sKlotho was positively associated with IGF1 $(P<0.0001)$ and height SDS $(P<0.001)$. Conclusion: The increase in serum phosphate, which we found in GH-deficient children under GH treatment, is not associated with suppression but rather than with upregulation of the phosphaturic FGF23/Klotho axis.
\end{abstract}

\section{Introduction}

Normal phosphate homeostasis is essential for appropriate bone and growth plate mineralization and therefore for normal linear growth. The bone-kidney endocrine axis mediated by the fibroblast growth factor 23 (FGF23) and Klotho has emerged as a major regulator of phosphate homeostasis; therefore, an intact FGF23/Klotho axis is important for normal linear growth. This becomes obvious in human genetic disorders with excess of FGF23 (hypophosphatemic rickets) (1) and Klotho (2) where linear growth is impaired.

FGF23 is a phosphaturic hormone that is predominantly produced in the bone by osteocytes and acts directly in renal phosphate handling in the proximal tubule, (c) 2016 European Society of Endocrinology Printed in Great Britain therefore promoting renal phosphate wasting. In addition, FGF23 causes hypophosphatemia via the inhibition of the $1 \alpha$-hydroxylase activity simultaneously with the stimulation of the 24-hydroxylase activity, leading to low serum levels of 1,25 -dihydroxyvitamin $\mathrm{D}\left(1,25-(\mathrm{OH})_{2}\right.$ vitamin $\left.\mathrm{D}\right)$ (3). FGF23 is catabolized by proteolytic cleavage to smaller $\mathrm{N}$-terminal and C-terminal fragments $(4,5)$. Its $\mathrm{N}$-terminal peptide binds to the FGF receptor (FGFR) in the tissues and its C-terminal to Klotho; thus, both terminals are participants in the activity of hormone (5).

A critical feature of FGF23 is that it requires Klotho, a single-pass transmembrane protein expressed in renal tubules, as an obligate co-receptor to bind and activate

Published by Bioscientifica Ltd. 
FGFRs (6). The clue to understand Klotho protein function was the fact that FGF23-deficient mice and Klothodeficient mice develop identical phenotypes consisting of premature-aging syndrome and hyperphosphatemia $(7,8)$.

On the other hand, the growth hormone (GH)/IGF1 axis, a major determinant of linear growth, has opposing effects on phosphate homeostasis than FGF23/Klotho system because it supports phosphate retention by increasing renal phosphate reabsorption $(9,10)$. In GH deficiency, phosphate tubular reabsorption is in the lower limit of normal or reduced, leading to a relative phosphate-deficient state. In addition, GH replacement increases renal phosphate reabsorption (TmPO4/GFR) and serum phosphate concentration (11), but the underline mechanisms remain unclear. One hypothesis is that GH has a direct anti-phosphaturic effect, and its principal product, IGF1, has been shown to increase TmPO4/GFR and 1,25-(OH $)_{2}$ vitamin $\mathrm{D}$ through a parathyroid hormone (PTH)-independent mechanism (9). GH/IGF1 could increase serum phosphate by suppressing FGF23/Klotho axis, but data in literature seem not supporting this hypothesis, since FGF23 and Klotho are increased in acromegaly with subsequent decrease after the removal of the adenomas $(12,13)$. In addition, in a few children with GH deficiency, GH treatment increased C-terminal FGF23 (14). However, it is not clear whether GH treatment is associated with increased intact FGF23, as that study measured the C-terminal form which assesses both proteolytic fragments and intact FGF23. The main objective of our study was to investigate the possible interactions of FGF23 and its co-receptor Klotho, with the GH/IGF1 axis in the regulation of phosphate metabolism in GH-deficient children after GH replacement therapy.

\section{Patients and methods}

\section{Patients}

In total, 23 children (13 girls and 10 boys) with confirmed GH deficiency participated in the study. The mean age at the start of treatment was 9.9 years (range 4.3-15.8). In total, 16 children were prepubertal (seven boys and nine girls) and seven children had entered puberty: four girls had Tanner stage III and three boys had testicular volume 8,12 and $20 \mathrm{ml}$.

All patients had decreased height velocity and height below the 3rd percentile ( -1.88 SDS). All children had classic idiopathic growth hormone deficiency (GHD), defined as two different GH provocation test peaks $<10 \mu \mathrm{g} / \mathrm{l}$ (Clonidine and L-Dopa stimulation tests) and
IGF1 below the mean of age- and sex-matched healthy controls. Bone age was delayed in all children by more than 2 years. All patients were treated with daily SC injections of biosynthetic human GH at a dose of $0.023 \mathrm{mg} / \mathrm{kg}$ per day.

Age, height (measured by a Harpenden stadiometer) and weight were measured, and pubertal status was clinically assessed according to Tanner stages. Height, weight and BMI were expressed as SDS of the Greek reference data which were automatically calculated by the software program Growth Analyzer 3.

The study was approved by the Ethics Committee of the University Hospital of Patras. All children and their parents were informed, and a written consent was obtained before participation in the study.

\section{Methods}

Anthropometry and assessment of biochemical parameters were performed before treatment and 3 months after the onset of GH treatment. Blood and urine samples were collected at $0800 \mathrm{~h}$ after overnight fasting for the assessment of serum phosphate, calcium, creatinine, alkaline phosphatase (ALP), IGF1, 1,25-(OH $)_{2}$ vitamin D, PTH, C-terminal and full length FGF23, soluble $\alpha$-Klotho (sKlotho), and urine creatinine, calcium and phosphate.

Biochemical analyses in serum (phosphate, calcium, ALP, creatinine) and urine (phosphate, calcium, creatinine) after overnight fasting were conducted using the automatic analyzer Olympus AU640 immediately after collection. Serum intact PTH was measured by chemiluminescence assay (Roche Elecsys, Roche Diagnostics). $1,25-(\mathrm{OH})_{2}$ vitamin D levels and IGF1 levels were measured by enzyme immunoassays (IDS GmbH, Frankfurt am Main, Germany).

The renal threshold for maximum tubular phosphate reabsorption rate (TmP/GFR; millimoles per liter of GFR) was calculated from values in serum and spot urine according to the nomogram by Walton and Bijvoet (15). FGF23 levels were determined by two different two-site enzyme-linked immunosorbent assays (ELISA), with an assay that measures only the active form of FGF23 which is the intact full-length FGF23 (iFGF23), in serum samples (Kainos Laboratories, Tokyo, Japan), and with another assay, the C-terminal assay (Immutopics C-terminal assay International, San Clemente, CA, USA) that measures both the active and the inactive FGF23 obtained after proteolytic cleavage of FGF23 (cFGF23) in EDTA plasma centrifuged straight away after collection. The Kainos intact assay uses two monoclonal antibodies to epitopes on either side of the cleavage site: thus, the assay only 
recognizes the intact molecule. The C-terminal assay uses polyclonal antiserum and recognizes two epitopes on the C-terminal side of the RXXR cleavage site and thus recognizes both full-length FGF23 and C-terminal cleavage fragments of FGF23. The intra- and inter-assay coefficient of variations for iFGF23 and CFGF23 were 2.3-3.6 and $3.1-4.2 \%$ respectively. Klotho was also determined by a sandwich ELISA with affinity purified antihuman Klotho (67G3) and antihuman Klotho (91F1) (IBL, Minneapolis, MN, USA) that measures sKlotho protein in human serum samples with an intra- and inter-assay coefficient of variations of 2.7 and $3.9 \%$ respectively. The antibodies used in this ELISA immunoprecipitate recombinant human sKlotho and a $130 \mathrm{kDa}$ protein from human plasma (16). The $130 \mathrm{kDa}$ form is produced by proteolytic cleavage of the glycosylated $135 \mathrm{kDa}$ full-length Klotho (17). All samples were stored at $-80{ }^{\circ} \mathrm{C}$ until analysis.

\section{Statistical analysis}

Statistical analysis was performed with SPSS 20 software for Windows. Data were expressed as mean \pm s.D. for parametric or median (range) for non-parametric data distribution. Differences in biochemical indices before and after GH treatment were analyzed by using the paired Student's $t$-test for parametric and Wilcoxon's paired test for non-parametric data distribution (Klotho and urine $\mathrm{Ca}$ ). For the comparisons in prepubertal or pubertal children were used non-parametric tests (Mann-Whitney and Wilcoxon) because of the small number of subjects. For univariate analysis, Pearson's correlation test was used for parametric and Spearman's correlation for non-parametric data distribution (the percentage (\%) of change in iFGF23 and Klotho). Partial correlations were performed with adjustment as appropriate for various parameters affecting the variables compared (age, sex and puberty). $P$ values $<0.05$ were considered as statistically significant.

\section{Results}

The height of the patients before GH treatment expressed as SDS was $-2.36 \pm 0.35$ S.D., and their height gain after 3 months on GH therapy was $0.12 \pm 0.08$ s.D. and after 6 months was $0.232 \pm 0.09$. The biochemical data of the $23 \mathrm{GH}$-deficient patients before and 3 months after GH treatment are summarized in Table 1 .

After 3 months on GH treatment serum phosphate, ALP levels and TmPO4/GFR were clearly elevated $(P<0.0001, \quad P<0.01$ and $P<0.01$ respectively), whereas serum calcium, PTH and $1,25-(\mathrm{OH})_{2}$ vitamin D
Table 1 Biochemical data before and 3 months after $\mathrm{GH}$ treatment. Data are expressed as mean \pm s.D. for parametric or median (range) for non-parametric data distribution (soluble $\alpha$-Klotho).

\begin{tabular}{|c|c|c|}
\hline & Before therapy & $\begin{array}{l}3 \text { months after } \\
\text { therapy }\end{array}$ \\
\hline Phosphate (mg/dl) & $4.65 \pm 0.39$ & $4.99 \pm 0.46 *$ \\
\hline Calcium (mg/dl) & $9.93 \pm 0.49$ & $9.99 \pm 0.38$ \\
\hline TmPO4/GFR & $5.30 \pm 0.90$ & $5.81 \pm 1.0^{\dagger}$ \\
\hline Alkaline phosphatase & 228 & $25 \overline{2} *$ \\
\hline PTH (pg/ml) & $41.88 \pm 11.26$ & $42.65 \pm 14.4$ \\
\hline $\begin{array}{l}1,25-(\mathrm{OH})_{2} \text { vitamin } \mathrm{D} \\
(\mathrm{pmol} / \mathrm{l})\end{array}$ & $116 \pm 28.59$ & $125 \pm 37.55$ \\
\hline $\begin{array}{l}\text { C-terminal FGF23 } \\
(\mathrm{RU} / \mathrm{ml})\end{array}$ & $50 \pm 15$ & $61 \pm 27^{\dagger}$ \\
\hline Intact FGF23 & $24.51 \pm 3.46$ & $43.81 \pm 5.28^{\ddagger}$ \\
\hline $\begin{array}{l}\text { Soluble } \alpha \text {-Klotho } \\
\text { (pg/ml) }\end{array}$ & $1340(433,7826)$ & $2485(515,7985)^{\ddagger}$ \\
\hline IGF1 (ng/ml) & $123 \pm 59$ & $204 \pm 83^{\ddagger}$ \\
\hline
\end{tabular}

did not change. The increase in serum phosphate was accompanied by a significant increase in plasma cFGF23 $(P<0.01)$, serum iFGF23 $(P<0.0001)$, sKlotho $(P<0.0001)$ and IGF1 $(P<0.0001)$ (Table 1) (Fig. 1$)$. The pubertal status did not affect the response to GH treatment, since in both prepubertal and pubertal children, ALP, serum phosphate, TmPO4/GFR, iFG23, sKlotho and IGF1 were increased, whereas serum calcium, PTH and 1,25-(OH $)_{2}$ vitamin $\mathrm{D}$ did not change. In prepubertal patients, cFGF23 was significantly increased after GH, but in pubertal patients, although it was increased, it did not reach statistical significance. Furthermore, the percentage of phosphate, cFGF23, iFGF23, sKlotho and IGF1 was similar in prepubertal and pubertal children (data not shown). The percentage of iFGF23 increase from baseline $(79 \pm 23 \%)$ was significantly higher than that of cFGF23 $(22 \pm 6 \%)(P<0.001)$.

Serum phosphate was positively related to TmPO4/GFR $(r=0.635 ; P<0.0001)$, iFGF23 $(r=0.427$; $P<0.01)$ and IGF1 $(r=0.385 ; P<0.05)$ when measurements before and after therapy were pulling together and adjusting for age, sex and puberty. TmPO4/GFR was positively correlated with iFGF23 $(r=0.314 ; P<0.05)$, IGF1 $(r=0.352 ; P<0.05)$, serum calcium $(r=0.337$; $P<0.05)$ and height $(r=0.348 ; P<0.05)$. The percentage of change (\%phosphate) in serum phosphate after GH treatment was negatively related to \%iFGF23 change $(r=-0.476 ; P<0.01)$ and positively to \%cFGF23 change $(r=0.501 ; P<0.01)$, whereas \%iFGF23 and \%cFGF23 changes were negatively related $(r=-0.448 ; P<0.05)$. 

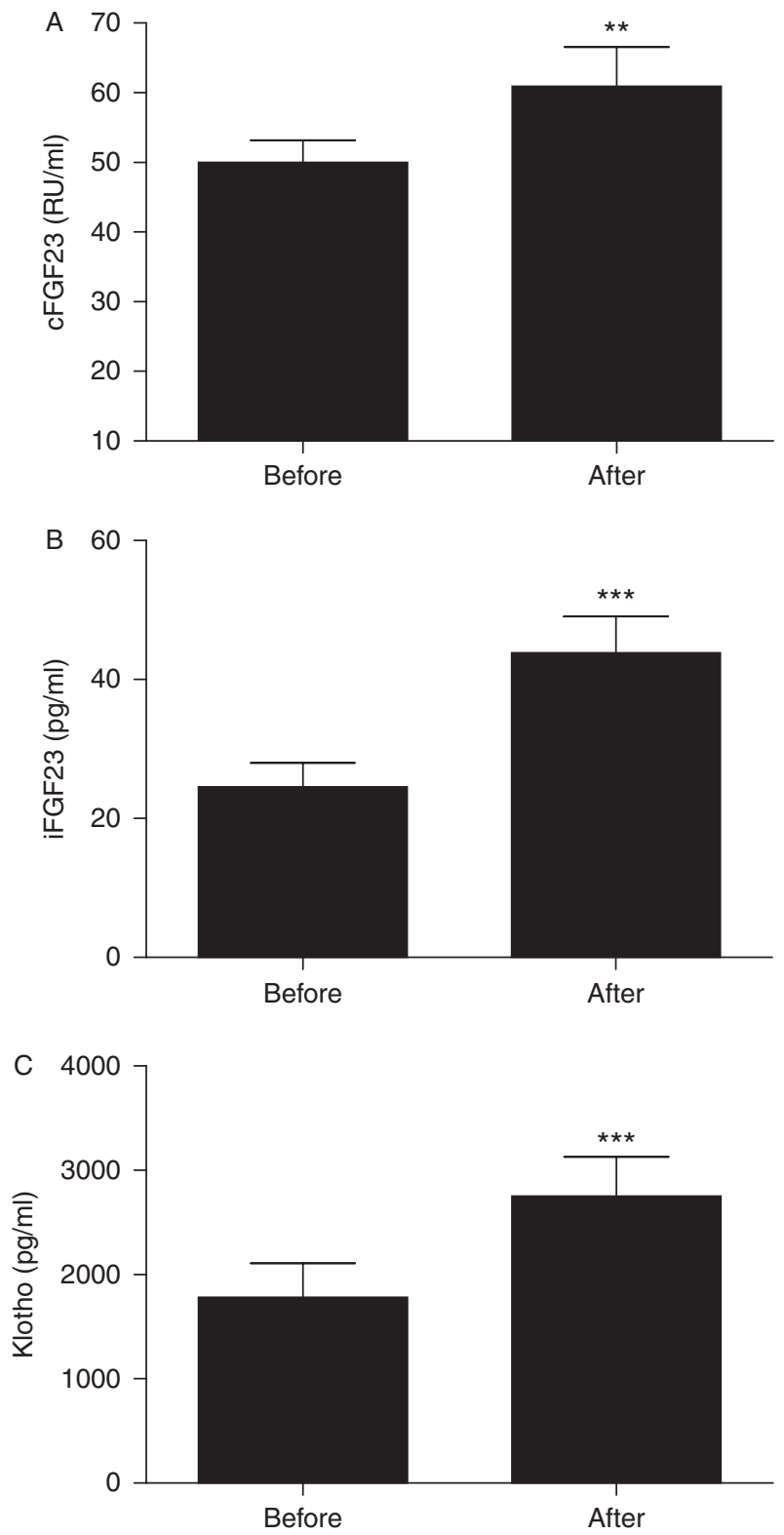

Figure 1

Serum levels of cFGF23 (A), iFGF23 (B) and Klotho (C) before and after 3 months of $\mathrm{GH}$ treatment in children with GHD.

$* * P<0.01 ; * * * P<0.0001$.

iFGF23 levels were positively correlated with sKlotho $(r=0.51 ; P<0.001)$, IGF1 ( $r=0.560 ; P<0.0001)$ (Fig. 2$)$ and height SDS $(r=0.543 ; P<0.0001)$ (Fig. 3$)$, and negatively with $1,25-(\mathrm{OH})_{2}$ vitamin $\mathrm{D}(r=-0.407 ; P<0.01)$. The \%iFGF23 was positively related to $1,25-(\mathrm{OH})_{2}$ vitamin D $(r=0.558 ; P<0.01)$. In contrast, cFGF23 was only associated with ALP $(r=0.449 ; P<0.01)$.
sKlotho was positively associated with IGF1 $(r=0.589$; $P<0.0001)$ (Fig. 2), height SDS $(r=0.467 ; P<0.001)$ (Fig. 3) and serum calcium $(r=0.318 ; P<0.05)$. The percentage of change (\%Klotho) in serum klotho was positively related to \%IGF1 change $(r=0.468 ; P<0.05)$.

Serum IGF1 levels were positively correlated with TmPO4/GFR $(r=0.4 ; P<0.01), 1,25-(\mathrm{OH})_{2}$ vitamin D $(r=0.330 ; P<0.05)$, serum calcium $(r=0.364 ; P<0.05)$, ALP $(r=0.393 ; P<0.01)$ and height SDS $(r=0.658 ; P<0.0001)$.

\section{Discussion}

In the present study, we investigated the effect of $\mathrm{GH}$ treatment on FGF23 and Klotho serum levels in GH-deficient children. We found that the increase in serum phosphate by GH treatment is accompanied by an increase in the phosphaturic FGF23/Klotho system.

We confirmed the effect of $\mathrm{GH}$ on phosphate metabolism, as there was a significant increase of serum
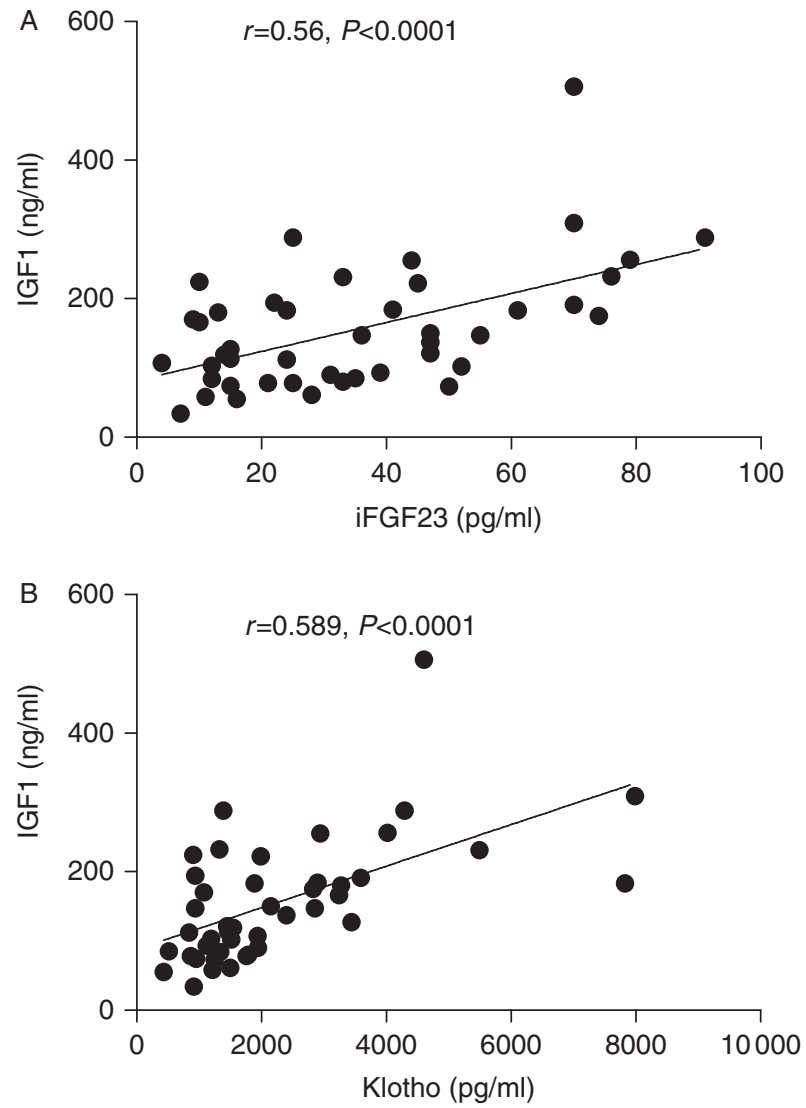

Figure 2

IGF1 relationship with iFGF23 (A) and Klotho (B) studied by partial correlation with adjustment for parameters affecting the variables (age, sex and puberty). 

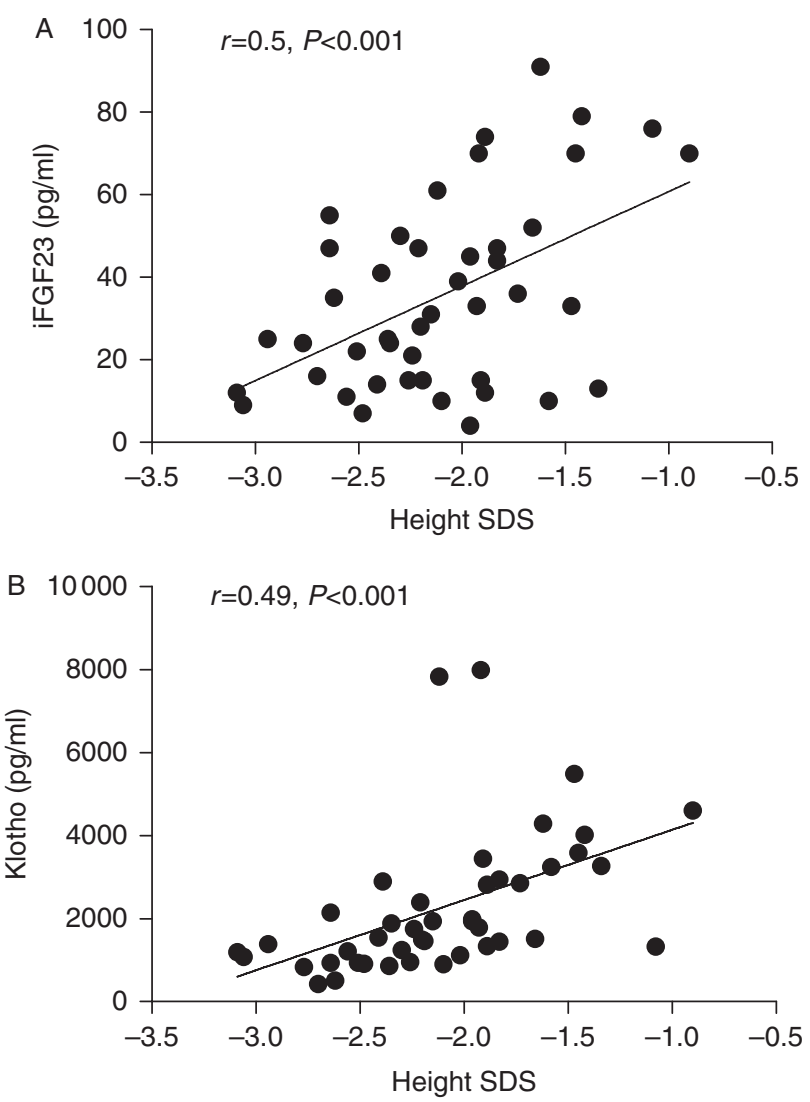

\section{Figure 3}

Height SDS relationship with iFGF23 (A) and Klotho (B) studied by partial correlation with adjustment for parameters affecting the variables (age, sex and puberty).

phosphate after 3 months of treatment resulting from a simultaneously significant increase in renal phosphate tubular reabsorption. Several studies in children and adults with GH deficiency have shown the same results of $\mathrm{GH}$ replacement on phosphate metabolism $(9,10,11,18)$. One could hypothesize that the GH/IGF1 axis increases serum phosphate by the suppression of the FGF23/Klotho system. However, our data clearly show that the increase in serum phosphate by $\mathrm{GH}$ is not mediated through the suppression of the phosphaturic FGF23/Klotho system. Instead, we observed an upregulation of both FGF23 and Klotho. We believe that $\mathrm{GH}$ treatment triggers the production of FGF23 as a feedback mechanism for the maintenance of serum phosphate homeostasis which is supported by the positive correlation between serum phosphate, renal phosphate tubular reabsorption and iFGF23 levels. Therefore, the effect of GH treatment on phosphate metabolism is mediated by another mechanism, such as a direct anti-phosphaturic effect of the GH/IGF1 axis which is well-supported in our study by the positive correlation of serum IGF1 with TmPO4/GFR or indirect effect through other unknown factors. However, it remains unclear whether the effect of the GH/IGF1 axis on the TmPO4/GFR is a GH alone or IGF1-driven effect (19).

Our results are in agreement with a similar report in GH-deficient children on GH treatment measuring only plasma cFGF23 levels (14). Interestingly, despite the higher doses of $\mathrm{GH}$ used in this study, almost double compared to our study, the magnitude of cFGF23 rise was similar indicating that the GH effect on cFGF23 is not dose dependent. From both studies, it is clear that the effect of GH on FGF23 is present from the 3rd month of GH therapy and lasts at least until the 8th month, but is unknown how early it appears and whether is sustained as long as GH treatment lasts. Furthermore, our data are supported by similar studies in patients with acromegaly. In this situation, the observed hyperphosphatemia is accompanied by increased levels of FGF23 (12) and Klotho (13) which are significantly decreased after the removal of the adenoma. Therefore, they also showed that the observed hyperphosphatemia is not caused by deficient FGF23/Klotho action.

In our study, we had a more intergraded approach, as we determined simultaneously cFGF23, iFGF23 and Klotho. We used these two different assays for FGF23, the intact Kainos and the C-terminal immutopic, because it has been referred to have the best correlation among other assays (20). Moreover, these two assays determine different fragments of the FGF23 peptide (intact FGF23 and intact FGF23 plus fragments respectively), which could provide a better understanding of the FGF23 physiology under GH treatment, since there are discrepant results in the literature investigating FGF23 with the C-terminal or the intact assay alone $(21,22,23)$. Furthermore, because of the aforementioned problems with the FGF23 commercially available assays, it is difficult to extrapolate the results from one to the other assay and from one to another study. More importantly, findings from studies in one pathological condition may not reflect the full aspects of FGF23 actions in phosphate homeostasis in another pathological condition or under normal circumstances. We found that iFGF23 was positively correlated to serum phosphate levels and TmPO4/GFR, finding which is in agreement with other studies in children $(24,25)$. On the other hand, our GHD patients with the highest \%phosphate increase had the lowest \%iFGF23 increase, since \%phosphate and \%iFGF23 were inversely related. This relationship is not contradictory to the serum phosphate 
and iFGF23 positive correlation, since the latter shows that iFGF23 increases according to serum phosphate, whereas the \%phosphate and \%iFGF23 correlation shows that the magnitude of the increase in serum phosphate is negatively related to the magnitude of the iFGF23 increase which is expected, since FGF23 has a phosphaturic action. Interestingly, the \%phosphate was positively related to the \%FGF23 increase in contrast to \%iFGF23. We do not have a direct explanation for this contradictory from the first glance finding, but it could indicate that as the \%phosphate becomes higher, FGF23 becomes more degraded. We based this assumption on the fact that iFGF23 assay determines only intact FGF23, whereas the cFGF23 assay determines both intact and cleaved FGF23, but in vitro studies are needed to investigate this assumption.

In addition to FGF23, serum Klotho was also significantly increased after $\mathrm{GH}$ treatment. This is not an unexpected finding, since Klotho controls FGF23 production in mice (26), acts as a co-receptor for FGF23 (6) and has phosphaturic action independent of FGF23 (27). Furthermore, in acromegaly, serum Klotho is dramatically increased and returned to normal levels after surgical removal of the GH-producing adenoma (13). Interestingly, we found a strong correlation between IGF1 and Klotho. We believe that this is not an incidental finding because data in literature support this relationship of IGF1 with the FGF23/Klotho axis (7) in healthy and in short stature children with sufficient and insufficient GH (28). So far, it is not delineated how Klotho and IGF1 are related and the exact mechanisms. There are indications in literature suggesting a role of Klotho in the regulation of the GH/IGF1 axis: Klotho-deficient mice are smaller than the wild type, and their somatotrophs have reduced number of secretory granules (8); Klotho inhibits IGF1 signaling $(29,30)$, and it enhances $\mathrm{GH}$ secretion in vitro likely through the suppression of the IGF1 signaling. Therefore, GH treatment could increase Klotho in order to suppress IGF1 signaling, since GH treatment increases the production of IGF1. On the other hand, because GH therapy increases serum phosphate, then the increase of Klotho, like the increase of FGF23, could be a counterregulatory reaction in order to keep serum phosphate levels within the normal limits. Again, similar to FGF23, the increase of serum Klotho after GH treatment is not known if it is a GH or IGF1 direct effect. The fact that even in non-GH-deficient children, IGF1 still correlates with Klotho (24) could support the assumption of an IGF1 rather than a GH direct effect on FGF23/Klotho axis.
As it was expected, height SDS was positively associated with IGF1, but more interestingly, it was also strongly associated with FGF23 and Klotho. The same association has been reported in normal children (7). This relationship should be seen through the fact that phosphate homeostasis is essential for linear growth and appropriate mineralization of the growth plate; therefore, an intact FGF23/Klotho axis is important. Human genetic disorders reveal the importance of FGF23 and Klotho in normal linear growth $(2,30)$. Thus, the positive relation we found between FGF23, Klotho and height SDS most likely reflects the significance of FGF23 and Klotho actions on linear growth.

In summary, we have found that the increase in serum phosphate in GH-deficient children under GH treatment is not associated with suppression but rather than with upregulation of the phosphaturic FGF23/Klotho axis. This most likely reflects a counter-regulatory mechanism in order to maintain a normal phosphate homeostasis. The strong association of Klotho with height SDS and IGF1 could indicate a role of Klotho in linear growth through the regulation of phosphate homeostasis, which is necessary for bone mineralization, or through other mechanisms. Further studies are needed to delineate the interplay between GH/IGF1 and FGF23/Klotho axis.

\section{Declaration of interest}

The authors declare that there is no conflict of interest that could be perceived as prejudicing the impartiality of the research reported.

\section{Funding}

This research did not receive any specific grant from any funding agency in the public, commercial or not-for-profit sector.

\section{References}

1 ADHR Consortium. Autosomal dominant hypophosphataemic rickets is associated with mutations in FGF23. Nature Genetics 200026 345-348. (doi:10.1038/81664)

2 Ichikawa S, Imel EA, Kreiter ML, Yu X, Mackenzie DS, Sorenson AH, Goetz R, Mohammadi M, White KE \& Econs MJ. A homozygous missense mutation in human KLOTHO causes severe tumoral calcinosis. Journal of Clinical Investigation 2007117 2684-2691. (doi:10.1172/JCI31330)

3 Fukumoto S. Physiological regulation and disorders of phosphate metabolism - pivotal role of fibroblast growth factor 23. Internal Medicine 200847 337-343. (doi:10.2169/internalmedicine.47.0730)

4 Shimada T, Mizutani S, Muto T, Yoneya T, Hino R, Takeda S, Takeuchi Y, Fujita T, Fukumoto S \& Yamashita T. Cloning and characterization of FGF23 as a causative factor of tumor-induced osteomalacia. PNAS 200198 6500-6505. (doi:10.1073/pnas.101545198)

5 Martin A, David V \& Quarles LD. Regulation and function of the FGF23/klotho endocrine pathways. Physiological Reviews 201292 131-155. (doi:10.1152/physrev.00002.2011) 
6 Kurosu $\mathrm{H} \&$ Kuro OM. The Klotho gene family as a regulator of endocrine fibroblast growth factors. Molecular Cellullar Endocrinology 2009299 72-78. (doi:10.1016/j.mce.2008.10.052)

7 Shimada T, Kakitani M, Yamazaki Y, Hasegawa H, Takeuchi Y, Fujita T, Fukumoto S, Tomizuka K \& Yamashita T. Targeted ablation of Fgf23 demonstrates an essential physiological role of FGF23 in phosphate and vitamin D metabolism. Journal of Clinical Investigation 2004113 561-568. (doi:10.1172/JCI200419081)

8 Kuro-o M, Matsumura Y, Aizawa H, Kawaguchi H, Suga T, Utsugi T, Ohyama Y, Kurabayashi M, Kaname T, Kume E et al. Mutation of the mouse klotho gene leads to a syndrome resembling ageing. Nature 1997 390 45-51. (doi:10.1038/36285)

9 Caverzasio J, Montessuit C \& Bonjour JP. Stimulatory effect of insulin-like growth factor-1 on renal Pi transport and plasma 1,25-dihydroxyvitamin D3. Endocrinology 1990127 453-459. (doi:10.1210/endo-127-1-453)

10 Kamenicky P, Mazziotti G, Lombes M, Giustina A \& Chanson P. Growth hormone, insulin-like growth factor-1, and the kidney: pathophysiological and clinical implications. Endocrine Review 201435 234-281. (doi:10.1210/er.2013-1071)

11 Nishiyama S, Ikuta M, Nakamura T, Tomoeda S \& Matsuda I. Renal handling of phosphate can predict height velocity during growth hormone therapy for short children. Journal of Clinical Endocrinology and Metabolism 199274 906-909. (doi:10.1210/jc.74.4.906)

12 Ito N, Fukumoto S, Taguchi M, Takeshita A, Takeuchi Y, Yamada S \& Fujita T. Fibroblast growth factor (FGF) 23 in patients with acromegaly. Endocrine Journal 200754 481-484. (doi:10.1507/endocrj.K06-217)

13 Sze L, Bernays RL, Zwimpfer C, Wiesli P, Brandle M \& Schmid C. Excessively high soluble Klotho in patients with acromegaly. Journal of Internal Medicine 2012 272 93-97. (doi:10.1111/j.1365-2796.2012.02542.x)

14 Gardner J, Ashraf A, You Z \& McCormick K. Changes in plasma FGF23 in growth hormone deficient children during rhGH therapy. Journal of Pediatric Endocrinology \& Metabolism 201124 645-650. (doi:10.1515/ JPEM.2011.301)

15 Walton RJ \& Bijvoet OL. Nomogram for derivation of renal threshold phosphate concentration. Lancet 19752 309-310. (doi:10.1016/S01406736(75)92736-1)

16 Yamazaki Y, Imura A, Urakawa I, Shimada T, Murakami J, Aono Y, Hasegawa H, Yamashita T, Nakatani K, Saito Y et al. Establishment of sandwich ELISA for soluble alpha-Klotho measurement: age-dependent change of soluble alpha-Klotho levels in healthy subjects. Biochemical and Biophysical Research Communications 2010398 513-518. (doi:10.1016/j.bbrc.2010.06.110)

17 Imura A, Iwano A, Tohyama O, Tsuji Y, Nozaki K, Hashimoto N, Fujimori T \& Nabeshima Y. Secreted Klotho protein in sera and CSF: implication for post-translational cleavage in release of Klotho protein from cell membrane. FASEB Letters 2004565 143-147. (doi:10.1016/j. febslet.2004.03.090)

18 Saggese G, Baroncelli GI, Bertelloni S, Cinquanta L \& Di Nero G. Effects of long-term treatment with growth hormone on bone and mineral metabolism in children with growth hormone deficiency. Journal of Pediatric 1993122 37-45. (doi:10.1016/S0022-3476(05)83484-5)
19 Bianda T, Hussain MA, Glatz Y, Bouillon R, Froesch ER \& Schmid C. Effects of short-term insulin-like growth factor-I or growth hormone treatment on bone turnover, renal phosphate reabsorption and 1,25 dihydroxyvitamin D3 production in healthy man. Journal of Internal Medicine 1997241 143-150. (doi:10.1046/j.1365-2796.1997.94101000.x)

20 Imel EA, Peacock M, Pitukcheewanont P, Heller HJ, Ward LM, Shulman D, Kassem M, Rackoff P, Zimering M, Dalkin A et al. Sensitivity of fibroblast growth factor 23 measurements in tumor-induced osteomalacia. Journal of Clinical Endocrinology and Metabolism 200691 2055-2061. (doi:10.1210/jc.2005-2105)

21 Bacchetta J, Cochat P, Salusky IB \& Wesseling-Perry K. Uric acid and IGF1 as possible determinants of FGF23 metabolism in children with normal renal function. Pediatric Nephrology 201227 1131-1138. (doi:10.1007/s00467-012-2110-3)

22 Smith ER, McMahon LP \& Holt SG. Method-specific differences in plasma fibroblast growth factor 23 measurement using four commercial ELISAs. Clinical Chemistry and Laboratory Medicine 201351 1971-1981. (doi:10.1515/cclm-2013-0208)

23 Smith ER, Cai MM, McMahon LP \& Holt SG. Biological variability of plasma intact and C-terminal FGF23 measurements. Journal of Clinical Endocrinology and Metabolism 201297 3357-3365. (doi:10.1210/jc.2012-1811)

24 Gkentzi D, Efthymiadou A, Kritikou D \& Chrysis D. Fibroblast growth factor 23 and Klotho serum levels in healthy children. Bone 201466 8-14. (doi:10.1016/j.bone.2014.05.012)

25 Fischer DC, Mischek A, Wolf S, Rahn A, Salweski B, Kundt G \& Haffner D. Paediatric reference values for the C-terminal fragment of fibroblast-growth factor-23, sclerostin, bone-specific alkaline phosphatase and isoform $5 \mathrm{~b}$ of tartrate-resistant acid phosphatase. Annals of Clinical Biochemistry 201249 546-553. (doi:10.1258/acb.2012.011274)

26 Smith RC, O’Bryan LM, Farrow EG, Summers LJ, Clinkenbeard EL, Roberts JL, Cass TA, Saha J, Broderick C, Ma YL et al. Circulating alphaKlotho influences phosphate handling by controlling FGF23 production. Journal of Clinical Investigation 2012122 4710-4715. (doi:10.1172/JCI64986)

27 Hu MC, Shi M, Zhang J, Pastor J, Nakatani T, Lanske B, Razzaque MS, Rosenblatt KP, Baum MG, Kuro-o M et al. Klotho: a novel phosphaturic substance acting as an autocrine enzyme in the renal proximal tubule. FASEB Journal 201024 3438-3450. (doi:10.1096/fj.10-154765)

28 Wolf I, Shahmoon S, Ben Ami M, Levy-Shraga Y, Mazor-Aronovitch K, Pinhas-Hamiel O, Yeshayahu Y, Hemi R, Kanety H, Rubinek T et al. Association between decreased klotho blood levels and organic growth hormone deficiency in children with growth impairment. PLOS ONE 20149 e107174. (doi:10.1371/journal.pone.0107174)

29 Wolf I, Levanon-Cohen S, Bose S, Ligumsky H, Sredni B, Kanety H, Kuro-o M, Karlan B, Kaufman B, Koeffler HP et al. Klotho: a tumor suppressor and a modulator of the IGF-1 and FGF pathways in human breast cancer. Oncogene 200827 7094-7105. (doi:10.1038/onc.2008.292)

30 Kurosu H, Yamamoto M, Clark JD, Pastor JV, Nandi A, Gurnani P, McGuinness OP, Chikuda H, Yamaguchi M, Kawaguchi H et al. Suppression of aging in mice by the hormone Klotho. Science 2005309 1829-1833. (doi:10.1126/science.1112766)

Received 14 October 2015

Revised version received 6 January 2016

Accepted 13 January 2016 